\title{
A NOTE ON UNIFORM PARACOMPACTNESS
}

\author{
MICHAEL D. RICE
}

\begin{abstract}
Three possible definitions for the paracompactness of a uniform space are presented and shown to be equivalent. A locally compact uniform space is shown to be uniformly paracompact if and only if it is uniformly locally compact. The set of points of a uniformly paracompact metric space that admit no compact neighborhood is shown to be compact; hence a metric topological group is uniformly paracompact if and only if it is locally compact.
\end{abstract}

It is well known that every metric space is paracompact, that is, each open cover of a metric space admits a locally finite open refinement [St]. In view of the natural uniformity associated with a metric space, it is natural to speculate whether metric spaces are, in a sense that requires definition, uniformly paracompact. In this note we investigate three possible definitions of uniform paracompactness. These definitions are shown to be equivalent and to represent surprisingly restrictive properties. In particular, in a group setting, uniform paracompactness is closely related to local compactness.

Throughout the paper we employ the basic properties of uniformities as developed in [I]; in particular, a (separated) uniformity consists of a family of uniform covers that satisfies certain axioms and generates a Hausdorff topology in a natural way. If $u$ denotes a uniformity, then $p u$ denotes the precompact uniformity having the basis of finite uniform covers which belong to $u$. If $u$ and $v$ are uniformities on the same set, then $u / v$ is the quasiuniformity generated by covers of the form $\left\{V_{s} \cap U_{t}^{s}\right\}$, where $\left\{V_{s}\right\} \in v$, and for each $s, Q^{s}=\left\{U_{t}^{s}\right\} \in u$. If $v$ has a basis of point-finite uniform covers, then $u / v$ is a uniformity [I, VII. 5]. Finally, $\alpha$ denotes the largest uniformity compatible (having the same topology) with the given topology; it has the basis of normal covers, or equivalently, of locally finite cozero covers.

Consider the following uniform-topological properties of a uniform space.

$(*)_{0}$ Each open cover $\mathcal{Q}$ admits a uniformly locally finite open refinement $\mathcal{U}^{\prime}$ (there exists a uniform cover, each of whose members meets at most finitely many members of $\left.\mathcal{Q}^{\prime}\right)$.

$(*)_{1}$ If $\mathscr{U}$ is an open cover, then $\mathscr{U}_{f}$ (the cover consisting of finite unions of the members of $\mathcal{Q}$ ) is a uniform cover.

Received by the editors September 23, 1975 and, in revised form, January 2, 1976 and March 24, 1976.

AMS (MOS) subject classifications (1970). Primary 54D20, 54E15; Secondary 54E35, 54E45.

Key words and phrases. Uniformly locally finite open cover, paracompact, uniformly paracompact, locally compact, uniformly locally compact. 
$(*)_{2}$ If $\mathcal{U}$ is an open cover, there exists a uniform cover $\widetilde{V}$ such that $\mathscr{U} / V$ has a finite subcover for each $V \in \mathcal{V}$.

REMARKS. (i) One easily shows that $(*)_{1}$ is equivalent to $(*)_{2}$ and that $(*)_{0}$ implies $(*)_{1}$.

(ii) If $u X$ satisfies $(*)_{1}$, then $u X$ is a complete uniform space. Let $\mathscr{F}$ be a Cauchy filter and choose an ultrafilter $\tilde{\mathscr{F}}$ that contains $\mathscr{F}$. It suffices to show that $\hat{\mathscr{F}}$ converges, for then $\mathscr{F}$ has a cluster point and hence converges. If $\hat{\mathscr{F}}$ does not converge, one may construct an open cover $\mathcal{V}=\left\{V_{s}\right\}$ such that $V_{s} \notin \hat{\mathscr{F}}$ for each $s$. Since $\hat{\mathscr{F}}$ is an ultrafilter, it follows that no member of the uniform cover $\mathfrak{V}_{f}$ belongs to $\hat{F}$, which contradicts that $\mathscr{F}$ is a Cauchy filter.

THeOREM 1. $u X$ satisfies $(*)_{1}$ if and only if $X$ is paracompact and $\alpha=p \alpha / u$.

Since each open cover of a paracompact space is normal, it is easily seen that the conditions imply $(*)_{2}$ and hence $(*)_{1}$. Conversely, to show that $X$ is paracompact, let $\mathcal{U}$ be an open cover. Since $\mathscr{U}_{f}$ is a uniform cover, there exists a locally finite open cover $\mathfrak{V}=\left\{V_{t}\right\}$ such that $\mathfrak{V}<\mathscr{Q}_{f}$ [I, I. 14]. For each $t$, choose a finite family $\left\{U_{s}: s \in F(t)\right\}$ from $\mathcal{Q}$ that covers $V_{t}$. Then $\left\{U_{s} \cap V_{t}: s\right.$ $\in F(t)\}$ is a locally finite open cover that refines $\mathcal{Q}$. To show $\alpha=p \alpha / u$, let 2 be an open cover and choose a closed uniform cover $\left\{G_{t}\right\}$ that refines $\mathcal{U}_{f}$. As above, choose a finite family $\left\{U_{s}: s \in F(t)\right\}$ from $\mathcal{O}$ that covers $G_{t}$; then $\mathcal{H}_{t}=\left\{U_{s}: s \in F(t)\right\} \cup\left\{X-G_{t}\right\}$ is a finite open cover that belongs to $p \alpha$ since $X$ is paracompact. Finally, $\left\{G_{t} \cap H: H \in \mathcal{H}_{t}\right\}$ is a member of $p \alpha / u$ that refines थ.

TheOrem 2. Properties $(*)_{0}$ and $(*)_{1}$ of a uniform space are equivalent.

Let $\mathcal{Q}$ be an open cover. Using Theorem 1, choose a locally finite open refinement $\mathcal{U}^{\prime}$ of $\mathcal{Q}$ and for each $p$ choose an open set $U p$ containing $p$ such that $U p$ meets at most finitely many members from $\mathcal{U}^{\prime}$. Set $\theta=\{U p\}$. Then $(*)_{1}$ implies $\theta_{f} \in u$ and since each member of $\theta_{f}$ meets at most finitely many members from $\mathcal{U}^{\prime}$, the proof is complete.

Based on the proof of Theorem 2, one sees that $\mathcal{Q}^{\prime}$ need not be required open in the definition of $(*)_{0}$.

THEOREM 3. ${ }^{1}$ uX satisfies $(*)_{0}$ if and only if each open cover is a member of $p u / u$.

If each open cover belongs to $p u / u$, then $\alpha=p \alpha / u$ and each open cover is normal, so by Theorems 1 and $2, u X$ satisfies $(*)_{0}$. To establish the converse, let $\mathcal{U}$ be an open cover of $X$. For each $x$, choose $U_{x} \in \mathcal{U}$ containing $x$ and a uniformly continuous function $X \stackrel{g_{x}}{\longrightarrow}[0,1]$ such that $g_{x}(x)=1$ and $g_{x} / X-U_{x}$ $\equiv 0$. Define $\mathscr{T}=\left\{g_{x}^{-1}\left(\frac{1}{2}, 1\right]\right\}$. Then $\mathscr{W}=\mathfrak{V}_{f}$ is a uniform cover. If $W$ $=\cup_{x \in F} g_{x}^{-1}\left(\frac{1}{2}, 1\right]$ betongs to $\mathscr{W}$, where $F$ is finite, define the cover $\mathbb{Q}_{W}$ $=\left\{g_{x}^{-1}(0,1]: x \in F\right\} \cup\left\{\cap_{x \in F} g_{x}^{-1}\left[0, \frac{1}{2}\right)\right\}$. Then $\mathbb{Q}_{W}$ is a finite uniform cover

\footnotetext{
1 The author would like to thank the referee for his simplification of the proof of Theorem 3 and for his strengthened restatement and proof of Theorem 5.
} 
(refined by $\wedge_{x \in F} g_{x}^{-1}(\delta)$, where $\delta$ is the uniform cover of $[0,1]$ consisting of spheres of radius $\frac{1}{2}$ ) and $\mathscr{Q}_{W / W}<\mathscr{Q}$; hence $\mathscr{U} \in p u / u$ and the proof of Theorem 3 is complete.

Remarks. (i) We note that Theorem 3 and [I, VII. 41] show that if $u X$ satisfies $(*)_{0}$, then $H(u X)$ (the hyperspace of $u X$ ) is complete.

(ii) Using Theorem 3 , it is easily shown that if $u X$ satisfies $(*)_{0}$, then each open cover may be subordinated by a partition of unity consisting of uniformly continuous functions.

(iii) Based on the preceding work, any one of the properties equivalent to $(*)_{0}$ might serve as a definition of uniform paracompactness.

THEOREM 4. A locally compact space is uniformly paracompact if and only if it is uniformly locally compact.

To prove the necessity, choose a uniform cover $\left\{K_{s}\right\}$ consisting of compact sets; then each open cover restricted to any $K_{s}$ has a finite subcover, so $(*)_{2}$, and hence by Theorem $2,(*)_{0}$ is satisfied. The proof of the sufficiency is left to the reader.

EXAMPLES. (i) A fine space is uniformly paracompact if and only if it is paracompact.

(ii) The hedgehog based on infinitely many copies of the unit interval is uniformly paracompact, but it is not locally compact.

(iii) From Theorem 4, a uniform space with discrete topology is uniformly paracompact if and only if there exists a uniform cover consisting of finite sets. Such a space need not be uniformly discrete. Let $M$ be the countable disjoint union of two point spaces $D_{n}$ with metric $d$ defined by $d(x, y)=1 / n$ if $x, y$ $\in D_{n}, x \neq y$, and $d(x, y)=1$ if $x, y$ belong to distinct $D_{n}$. Then $\left\{D_{n}\right\}$ is a uniform cover of $M$, but $M$ is not uniformly discrete.

(iv) The uniform product of two uniformly paracompact spaces need not be uniformly paracompact. Let $\alpha S$ be the fine space based on the Sorgenfrey line $S$; then by Theorem $1, \alpha S \times \alpha S$ is not uniformly paracompact since it is not paracompact. However, one may establish that uniform paracompactness is preserved by uniform sums, uniform quotients, and the formation of closed subspaces.

(v) A locally compact metric space need not be uniformly paracompact. For example, let $M=\cup \mathbf{N}_{n}$ be the countable disjoint union of the natural numbers with metric $d$ defined by $d(x, y)=1 / n$ if $x, y \in \mathbf{N}_{n}, x \neq y$, and $d(x, y)=1$ if $x, y$ belong to distinct $\mathbf{N}_{n}$. Clearly $M$ is topologically discrete, but if $\mathcal{Q}$ denotes the open cover consisting of singleton sets, then $\mathscr{U}_{f}$ is not a uniform cover. Note, however, that each open cover is a member of $d / d$ (compare Theorem 3) and may therefore be subordinated by a partition of unity consisting of uniformly continuous functions.

(vi) Let $X=\cup_{s<\Omega}\left\{0_{s}, 1_{s}\right\}$ be the uncountable disjoint union of two point spaces with the uniformity $u$ having as a basis the covers $\mathscr{Q}_{s}=\left\{\left\{0_{t}\right\},\left\{1_{t}\right\} ; t\right.$ $<s\} \cup\left\{\left\{0_{t}, 1_{t}\right\}: t \geqslant s\right\}, s<\Omega$. Then $u X$ is a measurable proximally fine 
space that is uniformly paracompact, but it is not locally fine.

(vii) Using example (v), one may show that a necessary condition for a countable product $\Pi X_{n}$ to be uniformly paracompact is that all but a finite number of the $X_{n}$ are compact. Note that by Remark (ii) preceding Theorem 1 , each $X_{n}$ is complete. Assume there exists an infinite subfamily $\left\{X_{i}\right\}$ of nonprecompact spaces. Then each $X_{i}$ contains a closed countably infinite uniformly discrete subset $N_{i}$ and $\Pi N_{i}$ is a closed subspace of $\Pi X_{i}$. Since the space $M$ found in example (v) may be uniformly embedded (in a closed manner) in $\Pi N_{i}$, it follows that $\Pi X_{i}$ is not uniformly paracompact, which is a contradiction. Hence, all but a finite number of the $X_{n}$ are compact. In particular, note that $\mathbf{N}^{\aleph_{0}}$ and $\mathbf{R}^{\aleph_{0}}$ are not uniformly paracompact.

THEOREM 5. The collection of points of a uniformly paracompact metric space that admit no compact neighborhood is compact.

Let $F$ be the collection of points of $X$ that admit no compact neighborhood. Clearly $F$ is closed, so if it is not compact there exists an infinite uniformly discrete sequence $\left\{x_{n}\right\} \subset F$. Let $\left\{F_{n}\right\}$ be a uniformly discrete sequence of closed neighborhoods of the respective points $x_{n}$ such that the diameter of $F_{n}$ is at most $1 / n$. For each $n$, choose a countable family $\mathcal{U}_{n}=\left\{U_{m}^{n}\right\}$ of open sets that covers $F_{n}$, but has no finite subcover (which is possible since $x_{n} \in F$ ), and define the cover $\mathcal{V}=\left\{U_{m}^{n}\right\} \cup\left\{X-\cup F_{n}\right\}$. Then $\mathcal{V}$ is an open cover of $X$, but $\mho_{f}$ is not a uniform cover.

COROllaRy 6. A metric group is uniformly paracompact if and only if it is locally compact.

Corollary 6 follows from Theorems 4 and 5 .

\section{REFERENCES}

[I] J. R. Isbell, Uniform spaces, Math Surveys, no. 12, Amer. Math. Soc., Providence, R. I., 1964. MR 30 \#561.

[R] M. D. Rice, Complete uniform spaces, TOPO 72-General Topology and Its Applications (Proc. 2nd Pittsburgh Internat. Conf., Carnegie-Mellon Univ. and Univ. of Pittsburgh, Pittsburgh, Pa., 1972), Lecture Notes in Math., vol. 378, Springer, Berlin, 1974. MR 50 \# 11166.

[So] R. H. Sorgenfrey, On the topological product of paracompact spaces, Bull. Amer. Math. Soc. 53 (1947), 631-632. MR 8, 594.

[St] A. H. Stone, Paracompactness and product spaces, Bull. Amer. Math. Soc. 54 (1948), 977-982. MR 10, 204.

Department of Mathematics, George Mason University, Fairfax, Virginia 22030

Current address: Bohušovicka 229, 19000 Praha 9, Czechoslovakia 